\title{
The SEMATECH Berkeley microfield exposure tool: learning at the 22-nm node and beyond
}

\author{
Patrick P. Naulleau, ${ }^{1}$ Christopher N. Anderson, ${ }^{2}$ Lorie-Mae Baclea-an, ${ }_{1}$ Paul Denham, ${ }^{1}$ \\ Simi Geroge, ${ }_{1}^{1}$ Kenneth A. Goldberg, ${ }^{1}$ Michael Goldstein, ${ }^{3}$ Brian Hoef, ${ }^{1}$ Russ Hudyma, ${ }^{4}$ \\ Gideon Jones, ${ }^{1}$ Chawon Koh, ${ }^{3}$ Bruno La Fontaine, ${ }^{5}$ Brittany McClinton, ${ }^{1}$ Ryan Miyakawa, ${ }^{1}$ Warren \\ Montgomery, John Roller, ${ }^{3}$ Tom Wallow, ${ }^{5}$ and Stefan Wurm ${ }^{3}$ \\ 'Center for X-Ray Optics, Lawrence Berkeley National Laboratory, Berkeley, CA 94720 \\ ${ }^{2}$ Applied Science \& Technology Dept., University of California, Berkeley, CA 94720 \\ ${ }^{3}$ SEMATECH, Albany, NY 12203 \\ ${ }^{4}$ Hyperion, San Ramon, CA 94582 \\ ${ }^{5}$ Advanced Micro Devices, Sunnyvale, CA 94088
}

\begin{abstract}
Microfield exposure tools (METs) continue to play a dominant role in the development of extreme ultraviolet (EUV) resists. One of these tools is the SEMATECH Berkeley 0.3-NA MET operating as a SEMATECH resist and mask test center. Here we present an update summarizing the latest resist test and characterization results. The relatively smail numerical aperture and limited illumination settings expected from 1st generation EUV production tools make resist resolution a critical issue even at the $32-\mathrm{nm}$ node. In this presentation, sub $22 \mathrm{~nm}$ half pitch imaging results of EUV resists are reported. We also present contact hole printing at the $30-\mathrm{nm}$ level. Although resist development has progressed relatively well in the areas of resolution and sensitivity, line-edge-roughness (LER) remains a significant concern. Here we present a summary of recent LER performance results and consider the effect of system-level contributors to the LER observed from the SEMATECH Berkeley microfield tool.
\end{abstract}

Keywords: extreme ultraviolet, lithography, photoresist, aberrations

\section{INTRODUCTION}

Microfield exposure tools (METs) [1-3] play a particularly important role in the area of extreme ultraviolet (EUV) resist development. This is due to the fact that the relative simplicity of such tools, in general, enables them to provide higher resolution capabilities than production scale alpha tools. For example, alpha tools now in use and preproduction tools in development $[4,5]$ have numerical apertures (NA) of 0.25 as compared to the NA of 0.3 available from the latest EUV METs. In the case of the SEMATECH Berkeley MET (BMET) [1,6,7], the higher NA is further enhanced by a unique programmable coherence illuminator allowing it to implement resolution enhancing illumination and achieve lower $k_{1}$ values (in principle, as low as 0.25). The SEMATECH Berkeley exposure tool utilizes a 5x-reduction, 0.3-NA optic designed and coated at Lawrence Livermore National Laboratory [8] and fabricated by Zeiss. It has a well-corrected field of view of $1 \times 3 \mathrm{~mm}$ at the reticle plane $(200 \times 600 \mu \mathrm{m}$ at the wafer plane). The CAD model shown in Fig. 1 depicts the major components of the exposure station as well as the EUV beam path (the system is described in detail in Ref. 1).

With EUV resists having reached resolution levels of $22 \mathrm{~nm}$ [9], the modified illumination capabilities [10] of the BMET are crucial to further progress. Figure 2 shows the computed aerial image contrast for the BMET as a function of illumination type. With conventional annular illumination, the contrast roll-off occurs at approximately 25 -nm half pitch. With the two $45^{\circ}$ dipole illuminations we can push the resolution below $22 \mathrm{~nm}$, and all the way down to $16 \mathrm{~nm}$ in one case, with relatively good linearity. Excellent performance at the 20 -nm level can also be achieved with the X dipole, however, not without forbidden pitches in the $25-\mathrm{nm} \mathrm{CD}$ and larger range. X dipole can be used to push the resolution beyond $16 \mathrm{~nm}$, but again with significant forbidden pitch effects. The forbidden pitch issue is a result of the central obscuration and depicted graphically in pupil space in Fig. 3. 


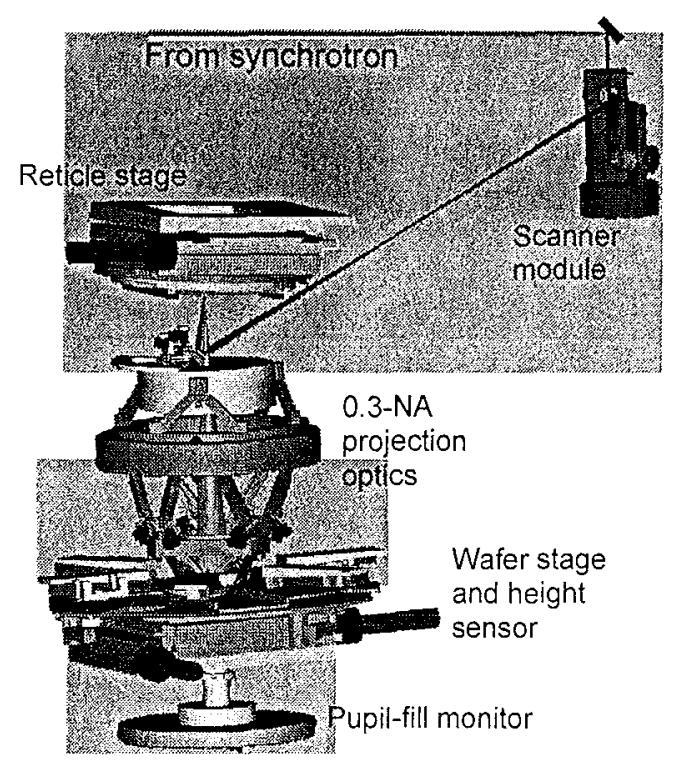

Fig. 1. CAD model of the Berkeley MET exposure tool.

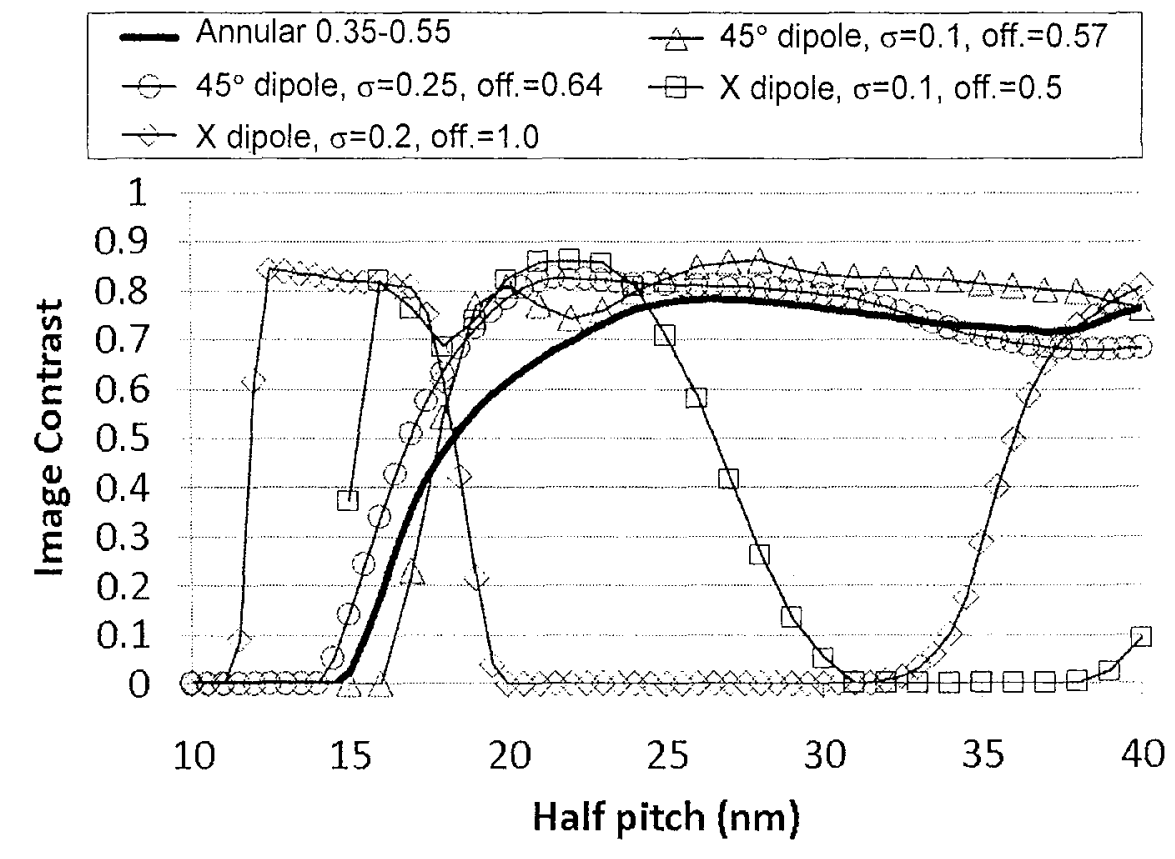

Fig. 2. Computed aerial image contrast for the BMET as a function of illumination type. 


\section{-1 diffracted}
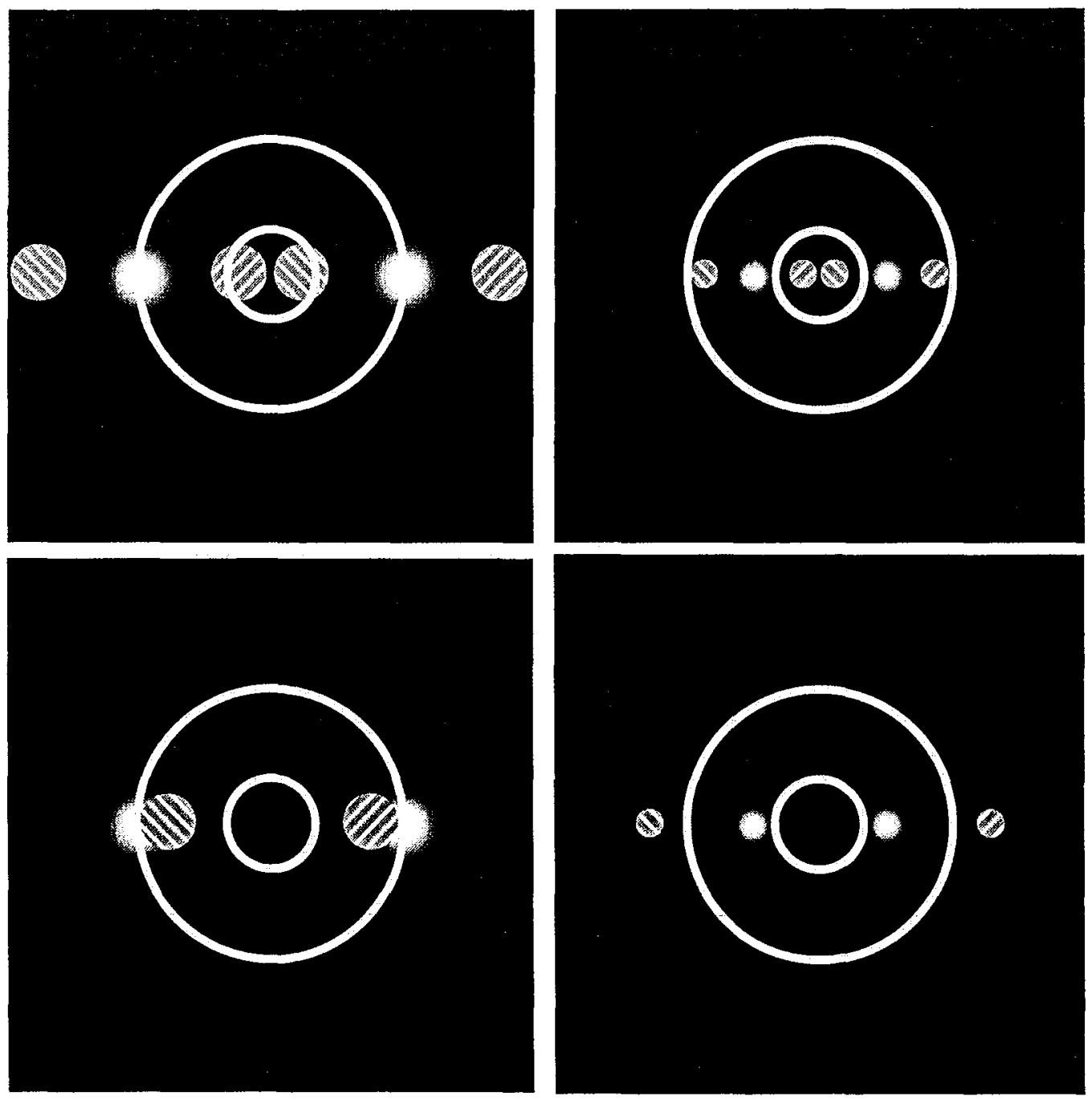

Fig. 3. Pupil source and diffraction diagrams for the two $X$ dipole pupil fills in Fig. 2. The top row assumes a 28-nm half-pitch object and the bottom row assumes a 13-nm half-pitch object. As long as at least one diffracted order falls within the annular pupil, the line space image can be generated. For 28 -nm features (top row), only the smaller offset dipole will produce an image, whereas for 13-nm features (bottom row) only the larger offset will work 


\section{RECENT RESIST TESTING RESULTS}

The past year has again brought about significant improvements in resist resolution. Figure 4 shows printing performance down to the 20-nm half-pitch level in a chemically amplified resist with a sensitivity of $15.2 \mathrm{~mJ} / \mathrm{cm}^{2}$ (sizing dose for $20-\mathrm{nm}$ features). Although the $20-\mathrm{nm}$ features are resolved, pattern collapse issues are evident. At $22 \mathrm{~nm}$, however, pattern collapse is not yet an issue. Note that the resist film thickness is $50 \mathrm{~nm}$, meaning that this resist supports an aspect ratio of slightly larger than $2: 1$ at $22-\mathrm{nm}$ feature sizes. Figure 4 also shows cross-section images demonstrating the fidelity of the process. The illumination settings are $45^{\circ}$ dipole with $\sigma=0.1$ and an offset of 0.57 .

The focus latitude of this resist is demonstrated in Fig. 5 where a depth of focus at best dose of approximately $150 \mathrm{~nm}$ is found for $22-\mathrm{nm}$ features. Also note the relatively stable through focus performance at 20 -nm half pitch despite the pattern collapse issues.

This same resist also performs extremely well in printing contacts. Figure 6 shows a cross-section of $30-\mathrm{nm} 1: 1$ contacts printed using annular illumination. The 30-nm contacts sized at a dose of $45 \mathrm{~mJ} / \mathrm{cm}^{2}$. Figure 7 shows the exposure latitude results for the 30 -nm contacts and Fig. 8 shows focus latitude results. An exposure latitude of $20 \%$ at best focus and depth of focus of $150-\mathrm{nm}$ at best dose is found. The exposure latitude is based on $\pm 10 \% \mathrm{CD}$ change and the depth of focus on setting an rms contact size variation limit of $2 \mathrm{~nm}$.
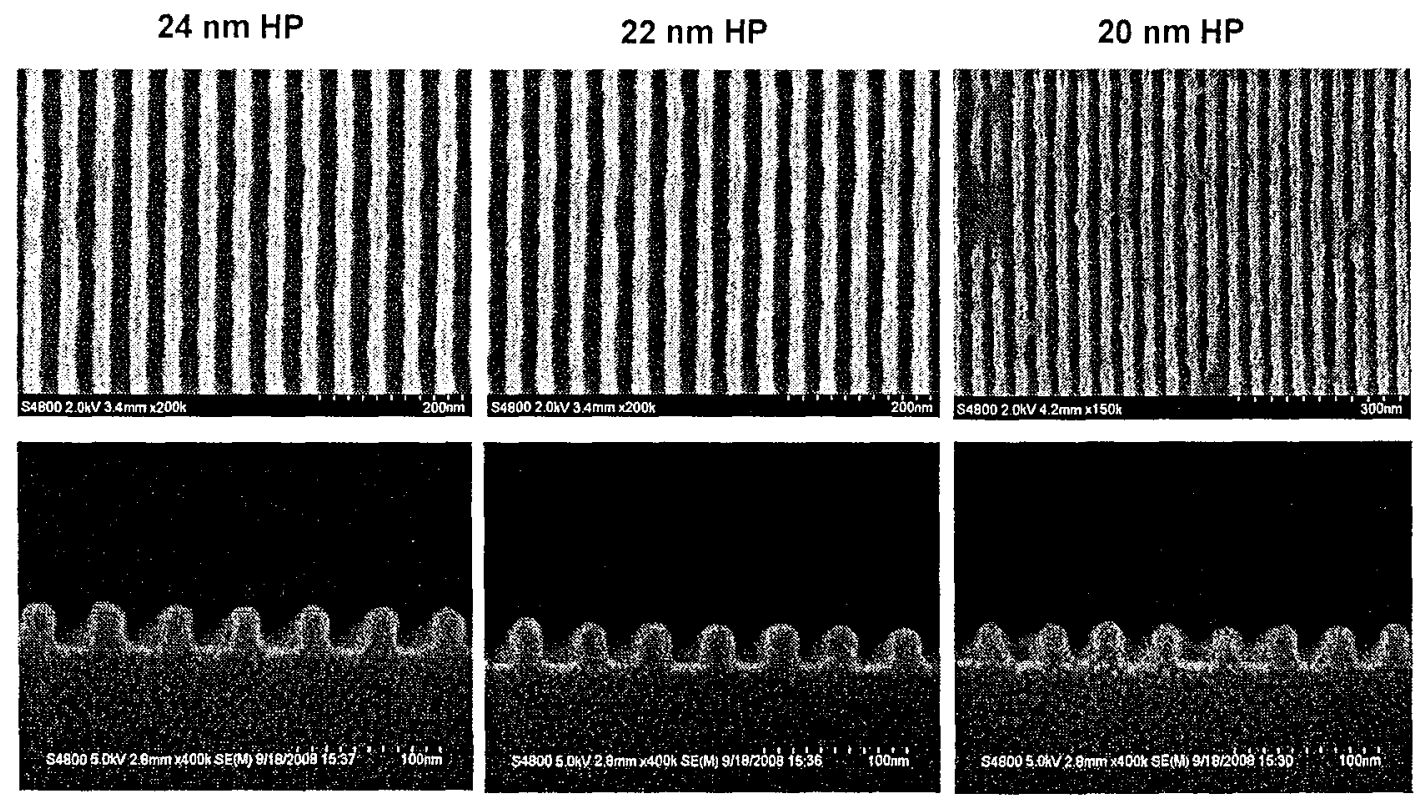

Fig. 4. Imaging results from a chemically amplified resist with a sizing dose $15.2 \mathrm{~mJ} / \mathrm{cm}^{2}$ for $20-\mathrm{nm}$ halfpitch features. The LER for $22-\mathrm{nm}$ half-pitch features is $3.9 \mathrm{~nm}$.

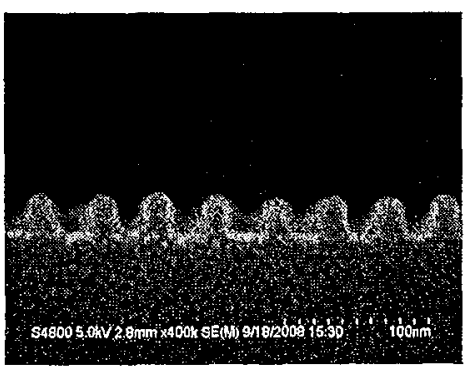




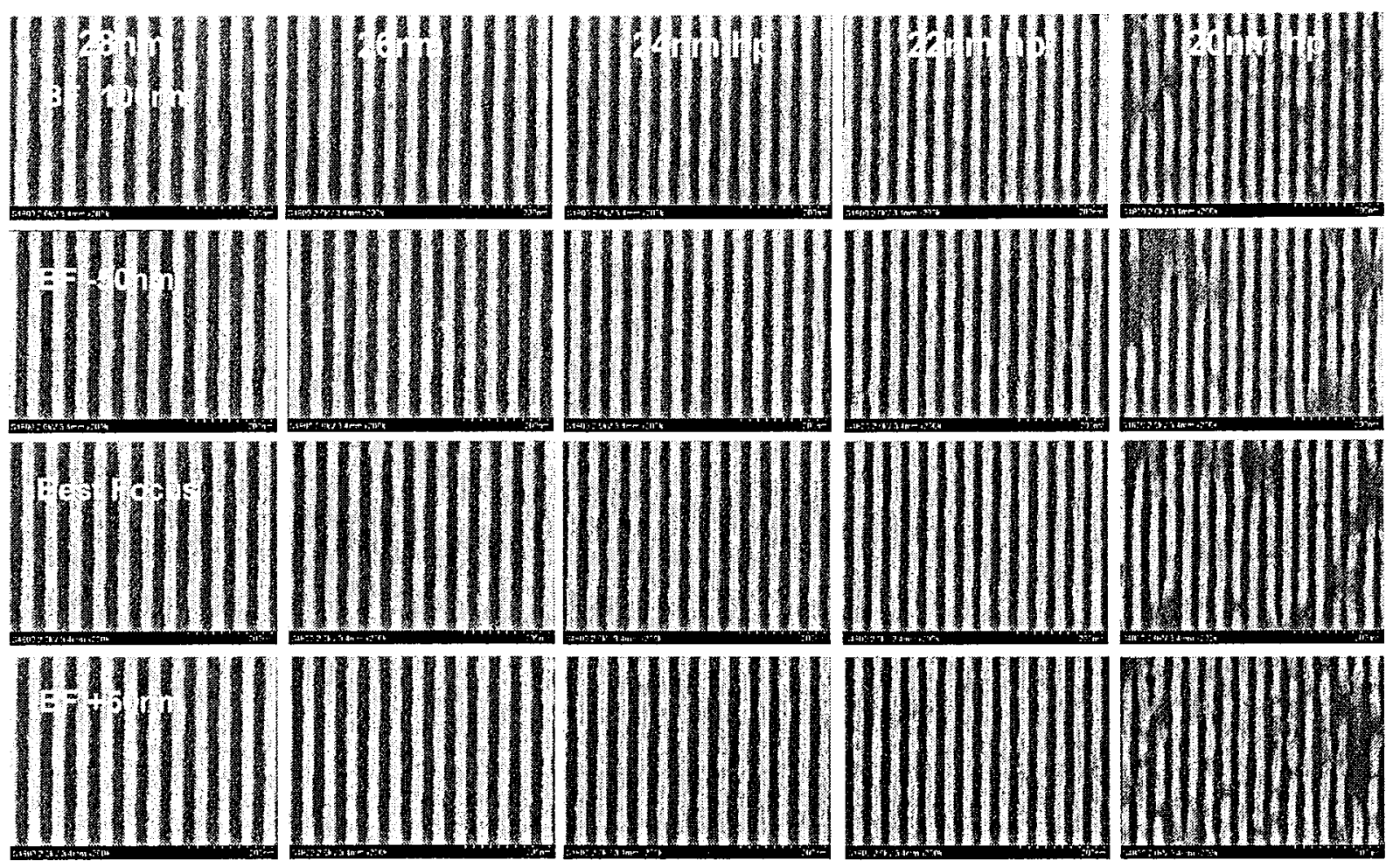

Fig. 5. Focus latitude results at best dose for the resist in Fig. 4.

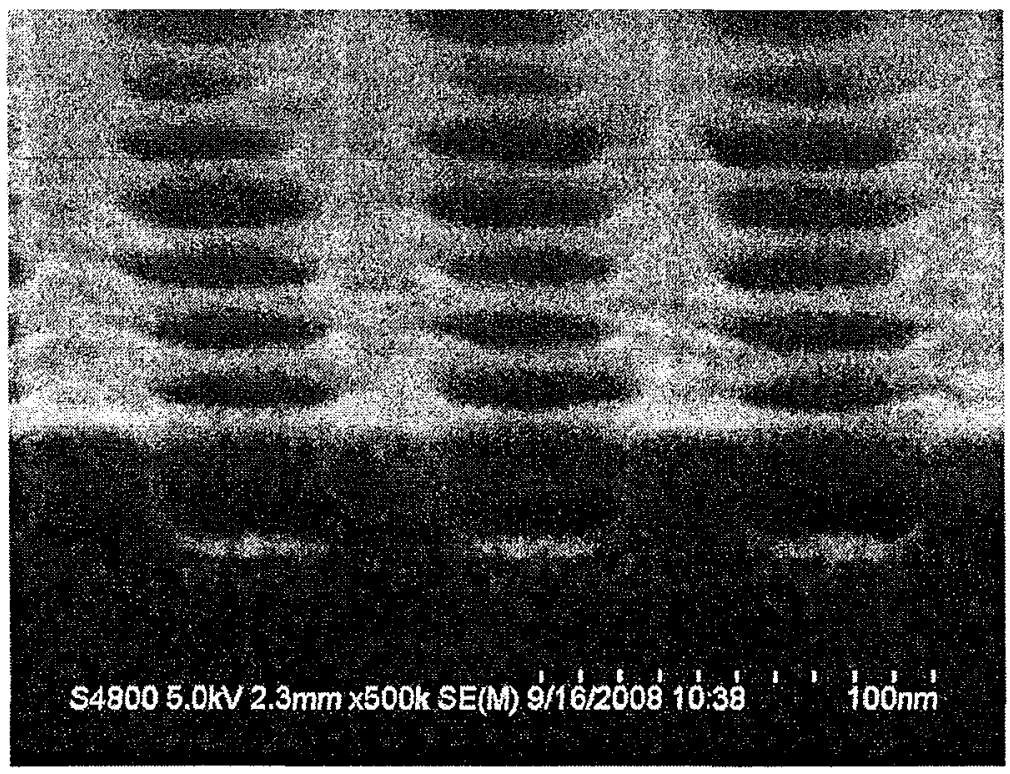

Fig. 6. 30-nm 1:1 contacts printed using annular illumination at a sizing dose of $45 \mathrm{~mJ} / \mathrm{cm}^{2}$. 

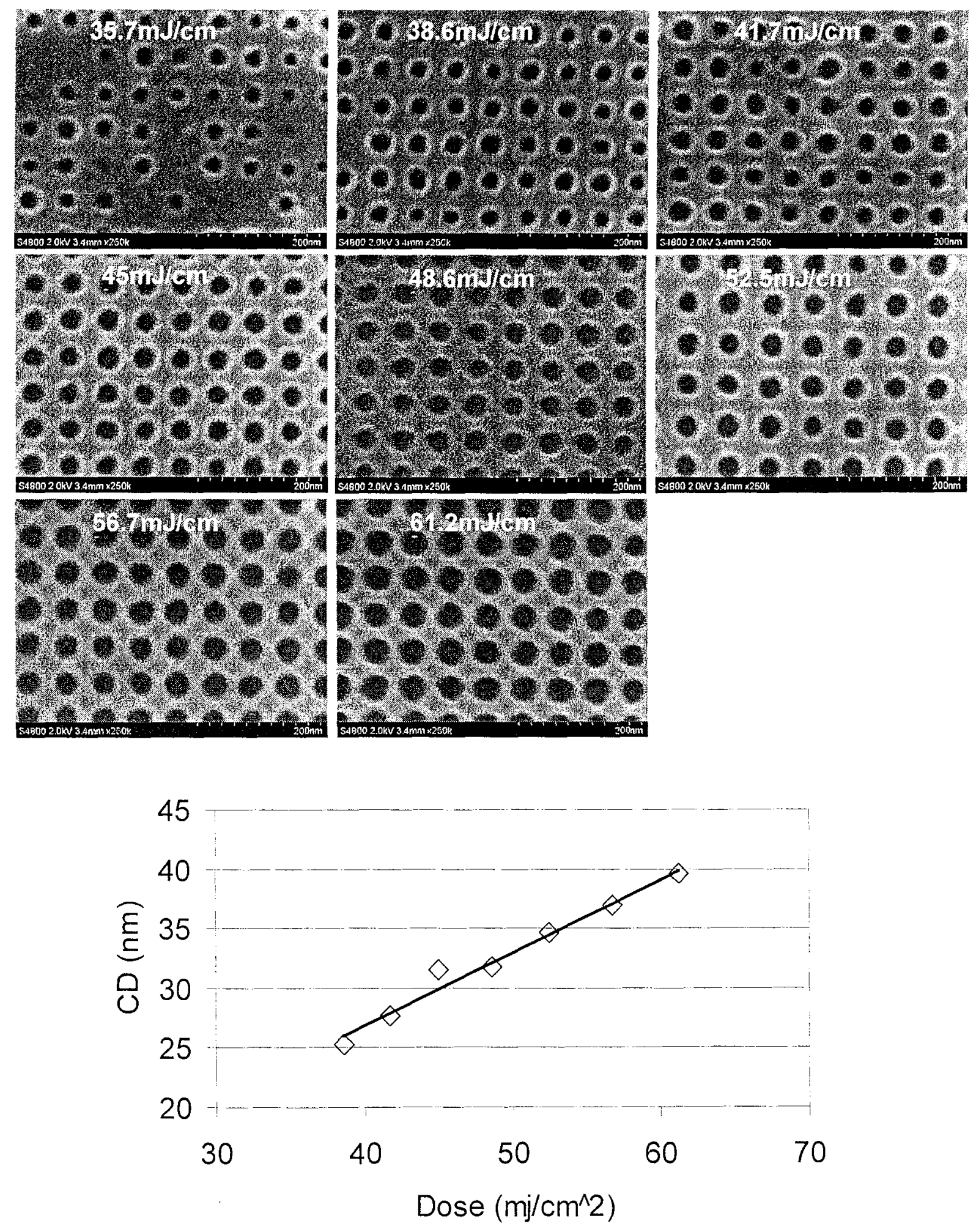

Fig. 7. 30-nm 1:1 contacts through dose. 

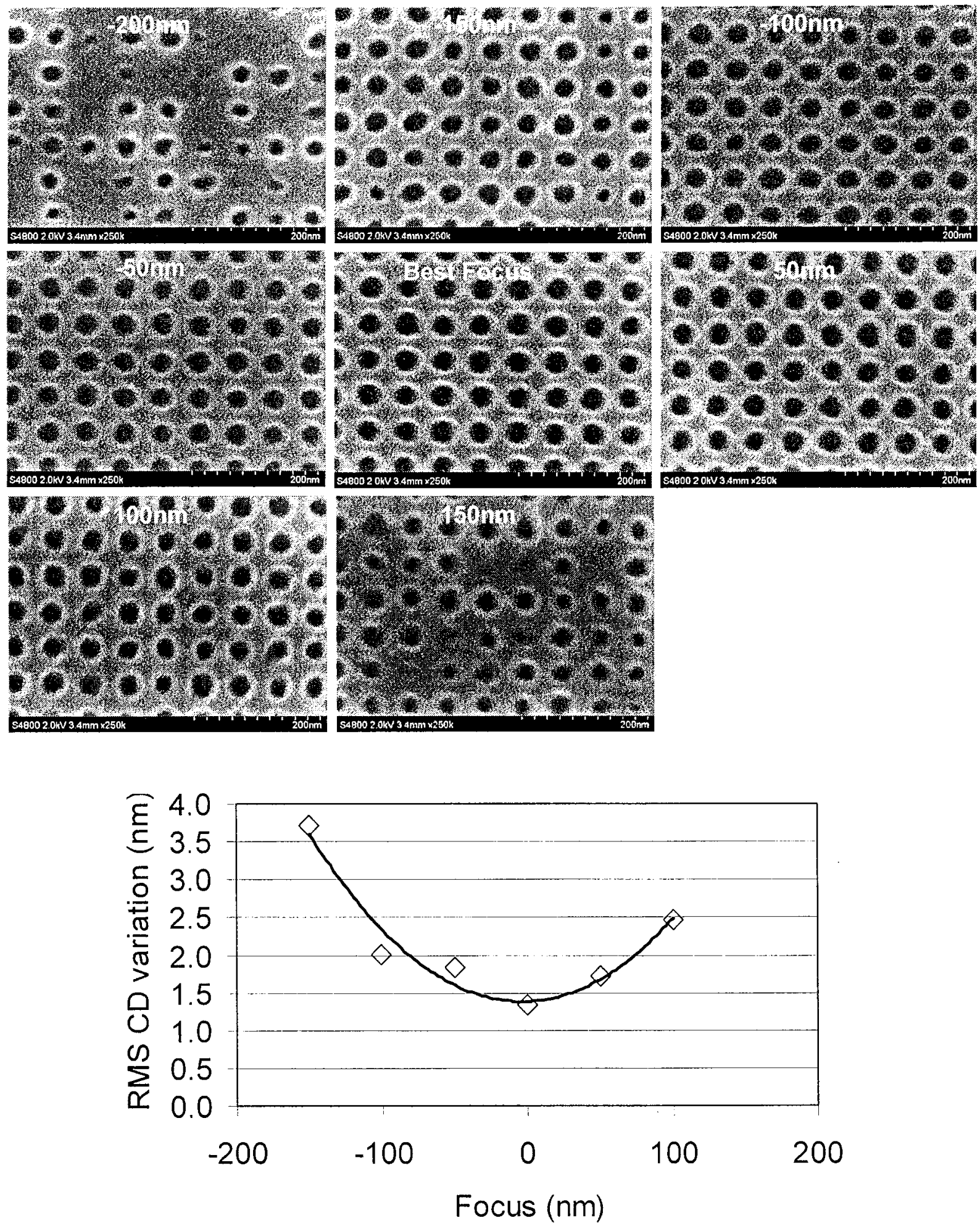

Fig. 8. $30-\mathrm{nm} 1: 1$ contacts through focus. 


\section{MEASUREMENT OF MASK INDUCED LER}

Despite excellent progress in the areas of resolution and sensitivity, LER remains a significant challenge. Modeling has shown, however, that the mask can be a significant contributor to image plane LER [11-15]. Here we present experimental results consistent with those modeling predictions. Mask-induced LER can come from two different sources: LER on the mask itself and mask reflector surface roughness (Fig. 9). These two sources are fundamentally different and respond differently to imaging parameters such as illumination coherence and defocus. The absorber LER is an obvious source and the extent to which it couples depends on the resolution of the imaging system. The Multilayer surface roughness couples to random phase roughness in the reflected field which depending on coherence and imaging characteristics becomes speckle (random intensity variations) in the image. This speckle, in turns, couples to LER. In general, the speckle problem gets worse with higher coherence and larger defocus. The absorber LER coupling is much less sensitive to illumination and defocus.

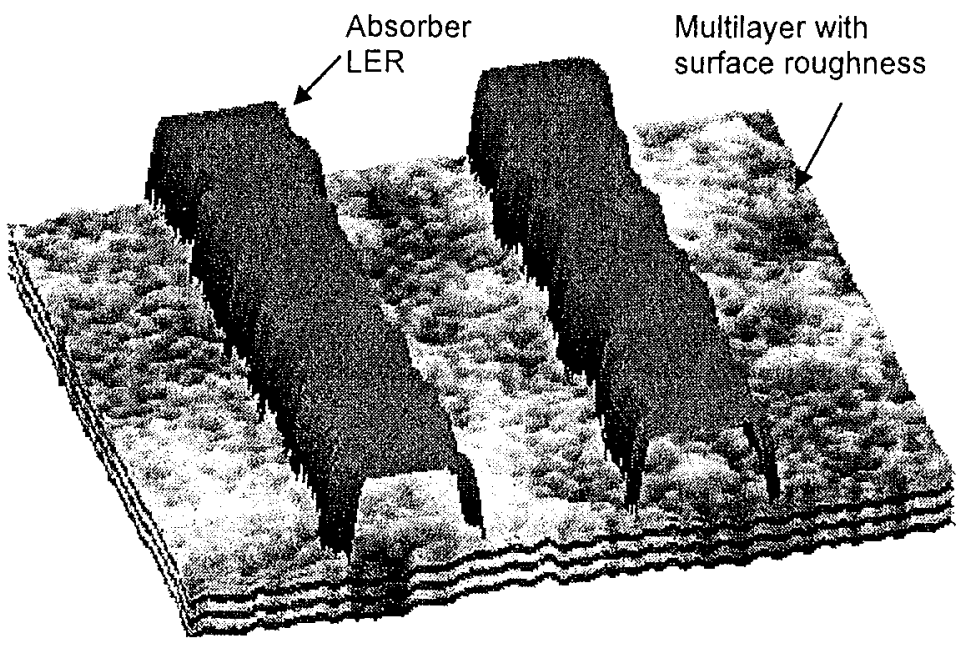

Fig. 9. Mask sources of image-plane LER.

To experimentally ascertain the importance of mask LER effects we have implemented a correlation measurement. Assuming that mask contributors to LER will be correlated from exposure to exposure and that resist and other random effects will not, the correlation measurement provides a means for distinguishing the two. Figure 10 shows three separate exposures of a specific line on the mask. The illumination setting is monopole with $\sigma=0.05$ and the wafer was intentionally positioned $100 \mathrm{~nm}$ out of focus. The high coherence and defocus were selected to accentuate multilayer roughness effects on the printed LER. Figure 11 shows a plot of the extracted lower edge [16] for the three independent images. A significant level of correlation is evident directly from the plot.

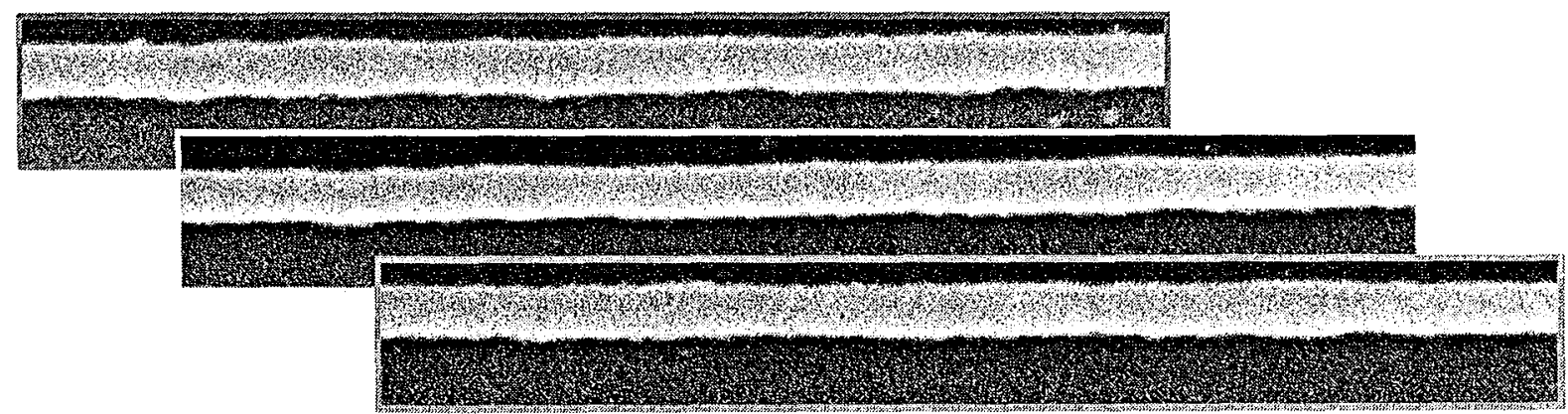

Fig. 10. Three independent exposures of a specific line on the mask. 


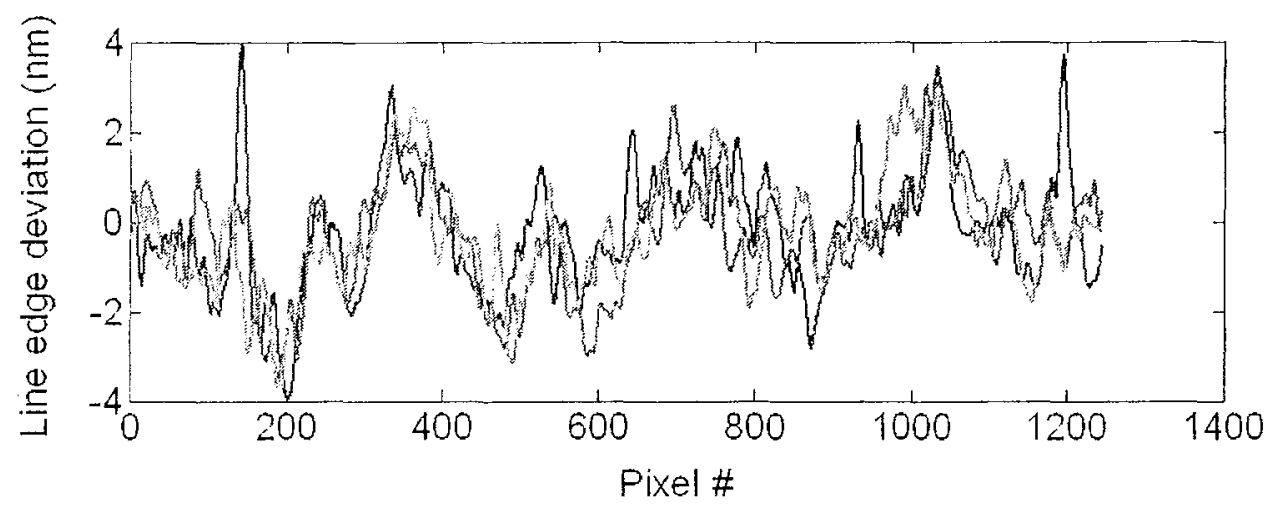

Fig. 11. Printed edge deviation plots for three independent exposures of a single line on the mask.

Using the line-edge data we find the average correlation to be $61 \%$. Noting that the total LER for this single edge is $4.3 \mathrm{~nm}$ on average, we find the correlated LER magnitude to be $3.4 \pm 0.2 \mathrm{~nm}$. The correlated LER is the full LER multiplied by the square root of the correlation. The uncertainty arises from the limited extent of the correlation measurement relative to the bandwidth of the roughness.

Next we compare the measured correlated LER to the predicted mask-induced LER under the same exposure conditions and the BMET mask parameters reported in the literature [14]. Figure 12 shows the computed binarized aerial image. The average LER measured from all the edges contained in the image is $3.0 \mathrm{~nm}$, very close to the measured correlated LER.

The measurements were repeated with lower coherence annular illumination and at best focus to minimize multilayer roughness effects yielding a measured correlation of $35 \%$ and a correlated LER magnitude of $2.0 \pm 0.3 \mathrm{~nm}$. Modeling under the same conditions yields a predicted mask-induced LER magnitude of $1.4 \mathrm{~nm}$.

The results show that mask effects on the printed LER are indeed measurable. Moreover, the measured correlated LER is in good agreement with predicted mask-induced LER values. The response of the correlated LER to coherence and defocus further suggest that mask multilayer roughness is a significant contributor.

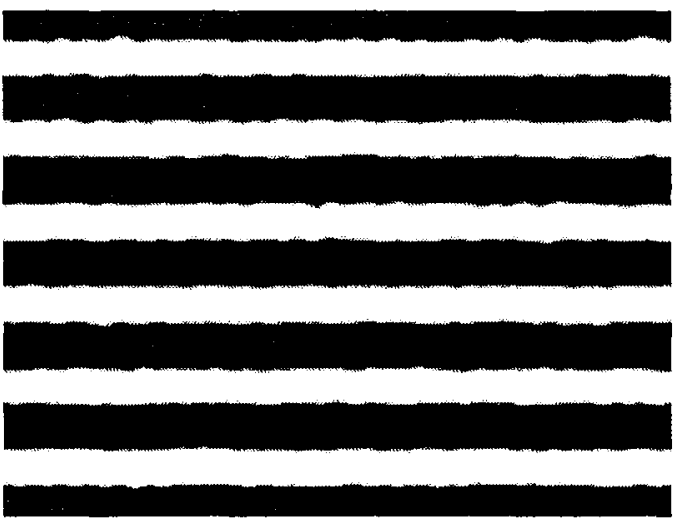

Fig. 12. Computed aerial image with monopole $\sigma=$ 0.05 illumination and $100-\mathrm{nm}$ wafer defocus. 


\section{FUTURE PLANS}

Although modified illumination can push the resolution of the 0.3 NA BMET down below $20 \mathrm{~nm}$, the benefits are restrictive in terms of pattern flexibility. More generalized EUV learning and development at the 16-nm node and below will require a higher NA system. To this end SEMATECH has initiated a plan to implement a $0.5 \mathrm{NA}$ microfield exposure tool at the Advanced Light Source synchrotron facility. A diagram of proposed two mirror optical system [17] is shown in Fig. 13. The system has a magnification of 5, a field of view of $200 \times 30 \mu \mathrm{m}$, and a mask angle of incidence of $6^{\circ}$. With modified illumination, the system has a resolution limit of $8 \mathrm{~nm}$, and with conventional illumination the system can easily resolve 16-nm features as depicted in Fig. 14.

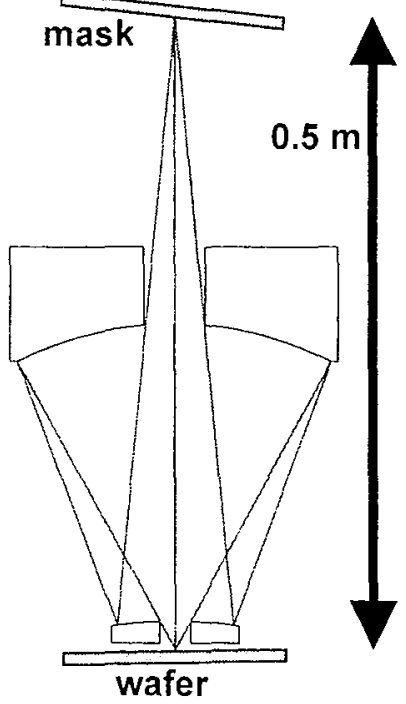

Fig. 13. Schematic of proposed 0.5-NA microfield lithography optic.

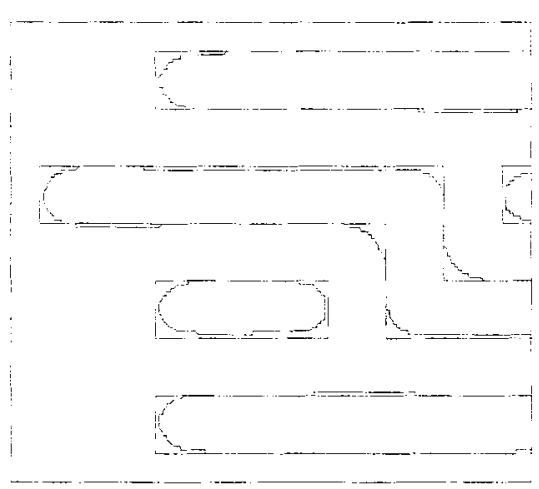

Fig. 14. Modeled performance of 0.5NA system in imaging 16-nm half pitch features with conventional illumination.

\section{SUMMARY}

As we enter the realm of sub-22-nm EUV development, resolution enhancing illumination capabilities are becoming increasingly important. Using dipole illumination, the SEMATECH Berkeley MET has demonstrated resolution down to the 20-nm half pitch level. The primary failure mechanism was pattern collapse. This resolution was achieved at a sensitivity of $15.2 \mathrm{~mJ} / \mathrm{cm}^{2}$ and the resist had an LER of $3.9 \mathrm{~nm}$ on $22-\mathrm{nm}$ half-pitch features. This same resist also supported contact resolution of $30 \mathrm{~nm}$ with $20 \%$ exposure latitude and $150-\mathrm{nm}$ depth of focus.

Experimental verification of predicted mask-induced roughness has been presented by way of exposure-to-exposure LER correlation measurements. The measured correlated LER values are in good agreement with predicted values based on known mask roughness characteristics.

Finally, to address the needs of EUV development at the 16-nm node and beyond, a new 0.5 -NA microfield exposure tool has been proposed. 
The authors are greatly indebted to the CXRO precision engineering team including Seno Rekawa, Kevin Bradley, Rene Delano, Drew Kemp, Jeff Gamsby, Bob Gunion, Ron Oort, Farhad Salmassi, and Ron Tackaberry for contributions in building and maintaining the tool. Moreover we thank Jim Thackeray of Rohm and Haas and Shinji Tarutani of Fujifilm for excellent resist support. This work was funded by SEMATECH and performed at Lawrence Berkeley National Laboratory using the SEMATECH MET exposure facility at the Advanced Light Source. Lawrence Berkeley National Laboratory's Advanced Light Source synchrotron facility is supported by the DOE, Office of Science, Basic Energy Sciences. Supported by the U.S. Department of Energy under Contract No. DE-AC02-05CH11231.

\section{REFERENCES}

1. P. Naulleau, et al., "Status of EUV micro-exposure capabilities at the ALS using the 0.3-NA MET optic," Proc. SPIE

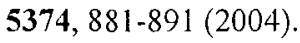

2. A. Brunton, et al., "High-resolution EUV imaging tools for resist exposure and aerial image monitoring," Proc. SPIE 5751, 78-89 (2005).

3. H. Oizumi, Y. Tanaka, I. Nishiyama, H. Kondo, K. Murakami, "Lithographic performance of high-numericalaperture (NA=0.3) EUV small-field exposure tool (HINA)," Proc. SPIE 5751, 102-109 (2005).

4. H. Meiling, et al., "First performance results of the ASML alpha demo tool," Proc. SPIE 6151, 615108 (2006).

5. M. Miura, K. Murakami, K. Suzuki, Y. Kohama, Y. Ohkubo, T. Asami, "Nikon EUVL development progress summary," Proc. SPIE 6151, 615105 (2006).

6. P. Naulleau, K. Goldberg, E. Anderson, K. Dean, P. Denham, J. Cain, B. Hoef, K. Jackson, "Characterization of the synchrotron-based 0.3 numerical aperture extreme ultraviolet microexposure tool at the Advanced Light Source," J. Vac. Sci. \& Technol. B 23, 2840-2843 (2005).

7. J. Cain, P. Naulleau, C. Spanos, "Resist-based measurement of the contrast transfer function in a 0.3-numerical aperture extreme ultraviolet microfield optic," J. Vac. Sci. \& Technol. B 24, 326-330 (2006).

8. R. Soufli, R. Hudyma, E. Spiller, E. Gullikson, M. Schmidt, J. Robinson, S. Baker, C. Walton, and S. Taylor, "Subdiffraction-limited multilayer coatings for the 0.3 numerical aperture micro-exposure tool for extreme ultraviolet lithography," Appl. Opt. 46, 3736-3746 (2007).

9. P. Naulleau, C. Anderson, J. Chiu, K. Dean, P. Denham, K. Goldberg, B. Hoef, S. Huh, G. Jones, B. La Fontaine, A. Ma, D. Niakoula, J. Park, T. Wallow, "Advanced extreme ultraviolet resist testing using the SEMATECH Berkeley 0.3-NA microfield exposure tool," Proc SPIE 6921, 69213N (2008).

10.P. Naulleau, K. Goldberg, P. Batson, J. Bokor, P. Denham, and S. Rekawa, "A Fourier-synthesis custom-coherence illuminator for EUV microfield lithography," Appl. Opt. 42, 820-826 (2003).

11.P. Naulleau and G. Gallatin, "The line-edge roughness transfer function and its application to determining mask effects in EUV resist characterization," Appl. Opt. 42, 3390-3397 (2003).

12. N. Beaudry, T. Milster, "Effects of mask roughness and condenser scattering in EUVL systems," Proc. SPIE. 3676, 653-662 (1999).

13.P. Naulleau, "The relevance of mask-roughness-induced printed line-edge roughness in recent and future EUV lithography tests," Appl. Opt. 43, 4025-4032 (2004).

14. P. Naulleau, D. Niakoula, G. Zhang, "System-level line-edge roughness limits in extreme ultraviolet lithography," J. Vac. Sci. \& Technol. B 26, 1289-1293 (2008).

15.P. Naulleau and G. Gallatin, "Spatial scaling metrics of mask-induced induced line-edge roughness," J. Vac. Sci. \& Technol. B, to be published (2008).

16. Line edge analysis was performed using the SuMMIT off-line LER analysis package.

17. Michael Goldstein, Russ Hudyma, Patrick Naulleau, Stefan Wurm, "Extreme-ultraviolet Microexposure Tool at 0.5 NA for Sub-16 nm Lithography," Opt. Lett. 33, 2995-2997 (2008). 